\title{
Valeur nutritionnelle des légumes feuilles consommés en Côte d'Ivoire
}

\author{
Anin Louise OCHO-ANIN ATCHIBRI ${ }^{1 *}$, Lêniféré Chantal SORO ${ }^{1}$, \\ Christophe KOUAME ${ }^{2}$, Edith Adouko AGBO ${ }^{1}$ et Kouakou Kouassi Armand KOUADIO ${ }^{1}$ \\ ${ }^{I}$ Université d'Abobo-Adjamé, UFR des Sciences et Technologies des Aliments (Côte d'Ivoire). \\ Laboratoire de Nutrition et de Sécurité Alimentaire (LANUSA) 02 BP 801 Abidjan 02. \\ ${ }^{2}$ Liaison Officer VBSS/AVRDC (Cameroun) P.O. Box 2008 MESSA. Yaoundé. \\ *Auteur correspondant, E-mail : aninatchibri@yahoo.fr; Tel: +22507 147677
}

\section{RÉSUMÉ}

La composition nutritionnelle de cinq légumes feuilles (Amaranthus hybridus, Celosia argentea, Corchorus olitorius, Cleome gynandra, solanum nigrum) collectés sur un site maraîcher (Port-Bouet) et deux marchés (Abobo-gare et Gouro) a été déterminée. Les résultats révèlent que les teneurs en nutriment des légumes feuilles sont plus élevées au niveau des marchés que sur le site maraîcher. Ainsi les feuilles sont riches en éléments minéraux avec des valeurs en potassium comprises entre 3034 et $22403 \mathrm{mg} / 100 \mathrm{~g}$ de MS. La teneur en calcium varie de 1546 à $5663 \mathrm{mg} / 100 \mathrm{~g}$ de MS, celle du phosphore varie de 1369 à $2410 \mathrm{mg} / 100 \mathrm{~g}$ de MS. Au niveau de la teneur en magnésium, elle est comprise entre 394 et $1947 \mathrm{mg} / 100 \mathrm{~g}$ de MS. La teneur en fer varie de 09 à $56 \mathrm{mg} / 100 \mathrm{~g}$ de MS. Les teneurs en protéines sont de plus de $25 \%$. Les légumes feuilles sont de bonnes sources de glucide (sucres totaux et réducteurs) avec des teneurs comprises entre 64 et $136 \mathrm{mg} /$ $100 \mathrm{~g}$ de MS pour les sucres réducteurs et 443 à $1381 \mathrm{mg} / 100 \mathrm{~g}$ de MS pour les sucres totaux. Cette étude montre que les légumes feuilles peuvent concourir au bien-être de l'organisme par leur apport en protéines et minéraux.

(C) 2012 International Formulae Group. All rights reserved.

Mots clés: Légumes-feuilles; valeur nutritionnelle; nutriments, post-récolte, Côte d'Ivoire

\section{INTRODUCTION}

Les légumes feuilles n'occupent pas une place de choix dans l'habitude alimentaire en Côte d'Ivoire. Ils sont cultivés comme plantes maraîchères et vendus sur les marchés. La consommation de ces légumes-feuilles est liée aux habitudes alimentaires des populations. Ainsi, sont consommés la corête potagère au Centre; l'amarante (Amaranthus hybridus), l'oseille de Guinée (Hibiscus sabdarifa), la morelle noire (Solanum nigrum) et les feuilles de patate au Nord (Ipomea batatas) ; la célosie (Celosia argentea), l'épinard (Spinach oleracea) et Caya blanc
(Cleome gynandra) à l'Ouest. Ces légumes feuilles renferment des micronutriments (vitamines, minéraux) qui contribuent au bienêtre de l'organisme (Iheanacho et al., 2009 ). Bien que ces légumes feuilles présentent de bonnes valeurs nutritionnelles (Tchiegang et al., 2004), ils sont souvent mal conditionnés avant d'être acheminés vers les marchés. Etant périssables par leur forte teneur en eau, ils sont sensibles aux actions des agents biologiques et physico-chimiques de dégradation. Il s'en suit des pertes post-récolte $\mathrm{du}$ point de vue quantitatif et qualitatif (Kahane et al., 2005). Cela suscite des 
inquiétudes concernant la qualité nutritionnelle de ces légumes feuilles vendus, surtout qu'ils ont une survie de $24 \mathrm{~h}$. En effet au niveau des marchés, il arrive que ces légumes feuilles ne soient pas vendus en une journée. Pour répondre à l'exigence du consommateur, les marchandes ont tendance à arroser ces légumes feuilles avec de l'eau, afin de garder leur fraîcheur. De ce fait il importe donc de vérifier la valeur nutritionnelle des légumes feuilles bord champ et post récolte vendus dans divers marchés d'Abidjan. C'est dans cette optique que la présente étude a été menée et s'est fixée comme objectif d'évaluer la valeur nutritionnelle des légumes feuilles. L'analyse des nutriments va nous permettre d'une part de comparer la valeur nutritive des légumes feuilles entre eux et d'autre part d'apprécier l'impact du site de collecte et de vente sur la qualité nutritive des légumes feuilles.

\section{MATERIEL ET METHODES \\ Matériel végétal}

Les légumes feuilles dont l'amarante (Amaranthus hybridus, Amaranthaceae), la célosie (Celosia argentea, Amaranthaceae), la corête potagère (Corchorus olitorius, Tiliacea), le caya blanc (Cleome gynandra, Capparaceae), et la morelle noire (solanum nigrum, Solanaceae) ont été collectés sur un site maraîcher (Port- Bouet), le marché Gouro (marché de gros) et le marché Abobo gare.

\section{Enquête}

L'enquête s'est déroulée dans deux marchés et un site maraîcher de production. Le choix des marchés s'est fait sur la base de l'origine des légumes feuilles et le site maraîcher sur le niveau de production. Concernant les marchés, le marché Gouro d'Adjamé constitue le marché de gros approvisionné par les sites maraîchers de la ville d'Abidjan, des villes environnantes et même de Bingerville. Quant aux deux autres marchés, l'approvisionnement se fait à partir du marché Gouro, de certains sites maraîchers situés dans les communes d'Abobo et de Yopougon, de quelques jardins familiaux environnants. Concernant le site maraîcher de Port Bouet, c'est le plus grand site maraîcher de la ville d'Abidjan vue sa superficie en comparaison avec celui du Banco dans la commune de Yopougon. S'agissant du choix des cinq légumes feuilles, il a été porté sur la fréquence de consommation et par le fait qu'ils sont peu connus. C'est le cas de l'amarantus hybridus, solanum nigrum, celosia argentea et le cleome gynandra. Nous notons également que les légumes feuilles sont récoltés tard la veille ou très tôt le matin avant d'être acheminés vers les marchés.

\section{Échantillonnage}

L'échantillonnage a eu lieu dans la ville d'Abidjan (Côte d'Ivoire). La collecte des échantillons s'est faite sur un site et les marchés au cours de la saison pluvieuse. Sur chaque site et marchés les légumes feuilles ont été prélevés auprès des producteurs et des vendeuses. Les variétés identiques ont été mises ensemble pour constituer un pool pour le site ou le marché. Les échantillons ont été mis dans une glacière contenant des carboglaces avant d'être acheminés au laboratoire pour l'analyse où chaque variété a été répartie en 3 lots de poids égal (ANONYME, 2001) pour constituer 3 répétitions. Pour chaque paramètre à déterminer, 3 mesures ont ainsi réalisées. La taille de l'échantillon était de 45.

\section{Analyse chimique}

La composition en sucres totaux et réducteurs des feuilles a été déterminée respectivement par la méthode de Dubois et al., (1956) et Bernfield, (1955). La teneur en protéine a été réalisée par la méthode de KJELDHAL (AOAC, 1984). La détermination de la teneur en minéraux a été effectuée au photomètre à flamme aux longueurs d'ondes respectives pour le Calcium (766,5 nm), Magnésium (422,7 nm), Potassium $(285,2 \mathrm{~nm})$. Le Fer a été déterminé au spectrophotomètre à $510 \mathrm{~nm}$ de longueur d'onde et la méthode molybdo-vanadate (AOAC, 2005) a été utilisée pour déterminer la quantité de phosphore. 


\section{Analyse statistique}

Une analyse statistique a été effectuée à partir du logiciel SPSS 16.0 for Windows. Le traitement des données s'est fait par une analyse de variance et les moyennes des paramètres de mesures (minéraux, protéines et sucres) ont été comparées par un test de Duncan au seuil $\alpha=0,05$.

\section{RESULTATS ET DISCUSSION}

Les données obtenues indiquent que les teneurs en sucres totaux sont plus élevées au niveau des marchés que celle du site maraîcher. Il y a une différence significative entre les teneurs en sucres totaux des légumes feuilles au seuil $\alpha=0,05$ (Tableau 1). L'amarante $(0,65 \pm 0,12 \mathrm{~g} / 100 \mathrm{~g}$ de MS), la corête Potagère $(1,16 \pm 0,46 \mathrm{~g} / 100 \mathrm{~g}$ de MS), la célosie $(0,83 \pm 0,31 \mathrm{~g} / 100 \mathrm{~g}$ de MS $) \mathrm{du}$ marché Gouro et la morelle Noire $(1,38 \pm 0,02$ $\mathrm{g} / 100 \mathrm{~g}$ de MS), le caya blanc $(0,73 \pm 0,02 \mathrm{~g}$ /100 g de MS) du marché abobo-gare ont les teneurs en sucres totaux élevées que ceux du site maraîcher. Concernant la teneur en sucres réducteurs, les légumes feuilles du site maraîcher ont les teneurs les plus élevées et significativement différents (Tableau 2). Les sucres réducteurs varient de 0,09 $\pm 0,01 \mathrm{~g} /$ $100 \mathrm{~g}$ de MS (Morelle Noire) à 0,81 $\pm 0,01 \mathrm{~g} /$ $100 \mathrm{~g}$ de MS (caya blanc) pour les légumes feuilles du site maraîcher contre 0,07 $\pm 0,01 \mathrm{~g}$ / 100 g de MS (amarante, morelle Noire, célosie du marché Gouro, et amarante et célosie du marché Abobo gare) à 0,26 $\pm 0,01$ g /100 g de MS (corête Potagère du marché Gouro) pour les marchés.

Les valeurs de sucres totaux et réducteurs du Corchorus olitorius des différents sites (marchés et site maraîcher) est faible par rapport à celle trouvée par Tchiegang et Kitikil (2004) (sucres totaux = $43,35 \pm 0,00 \mathrm{~g}$ et sucres réducteurs $=39,76 \pm$ $0,00 \mathrm{~g}$ ). Les différences entre les teneurs en sucres totaux et réducteurs des légumes feuilles pourraient être liée à la nature des feuilles, mais aussi à des problèmes d'ordre méthodologique, car les méthodes de dosage des sucres sont nombreuses et différentes selon la nature de l'oxydant ou selon les conditions du protocole expérimental (Audigie et al., 1977).
L'analyse statistique des teneurs en protéine montre qu'ils sont significativement différents au seuil de 5\%. Les taux sont plus élevés au niveau des marchés que ceux du site maraîcher. L'amarante $(37,21 \%)$, la corête potagère $(32,18 \%)$ et la célosie $(34,25 \%)$ du marché Abobo Gare, la morelle Noire $(37,5 \%)$ du marché Gouro ont les concentrations plus élevées que celles du site (Tableau 3). Néanmoins le caya blanc $(37,17 \%)$ du site a la teneur la plus élevée comparativement à celui des marchés. Les données obtenues indiquent également que les légumes feuilles sont une bonne source de protéine. En effet, Sheetal et al. (2005) ont montré que les légumes feuilles tropicaux sont riches en protéines et peuvent contribuer à assurer la sécurité alimentaire des populations pauvres. Ils peuvent aussi constituer de par leur composition, un complément appréciable de calories, de vitamines, de fibres, de sels minéraux et de protéines dans l'alimentation (Jansen et al., 2004). L'utilisation de l'engrais azoté lors de la production influence la teneur en protéine (Agbo et al., 2009).

Tous les légumes feuilles contiennent un taux élevé en minéraux. Les teneurs en phosphore de l'amarante $(2050 \mathrm{mg} / 100 \mathrm{~g}$ de MS), la corête potagère (2262 mg / 100 g de MS) et la célosie (1772 mg / 100 g de MS) du site maraîcher sont plus élevées que celles des marchés et diffèrent significativement (Tableau 4). Les teneurs en phosphore de la morelle Noire (2229 mg / 100 g de MS), le caya blanc (2410 mg / $100 \mathrm{~g}$ de MS) du marché Abobo gare sont élevées que celles du marché Gouro et site maraîcher.

Nos valeurs en minéraux diffèrent de celles obtenues par Grubben et al. (2004) qui montrent beaucoup de différences (corête = $122 \mathrm{mg}$, célosie = $43 \mathrm{mg}$, amarante $66 \mathrm{mg}$, caya blanc $=111 \mathrm{mg}$ et morelle $=75 \mathrm{mg}$ ). Cependant les variations dans la composition minérale sont influencées par les pratiques culturales (Nordeide et al., 1996 ; Agbo et al., 2009).

Les teneurs en calcium des légumes feuilles des marchés (5663 mg / $100 \mathrm{~g}$ de MS de l'amarante du marché Gouro, 2260 mg / 
$100 \mathrm{~g}$ de MS de la morelle Noire et $4927 \mathrm{mg} /$ 100 g de MS pour le caya blanc du marché Abobo-gare) sont plus élevées que celles du site maraîcher et sont significativement différentes (Tableau 5). La teneur en calcium de la célosie du site et du marché Gouro ne diffère pas significativement. Les données en calcium des légumes feuilles analysés dont l'amarante du site (2893 mg / 100 g de MS), la morelle du marché Abobo gare $(2260 \mathrm{mg} /$ $100 \mathrm{~g}$ de MS) est proche de celle trouvée par Odhav et al. (2007) (2363 mg et $2067 \mathrm{mg}$ ) respectivement. Les légumes feuilles du marché abobo gare ont les teneurs en calcium élevées. Ces concentrations pourraient être liées à l'origine diverse (champs, autres sites maraîchers et jardins) des légumes feuilles. Les légumes feuilles étudiés ont une bonne valeur en calcium. Cela est essentiel car le calcium est un facteur majeur dans l'ossification des os, joue un rôle dans la contraction musculaire, et l'absorption de la vitamine B12 (Mensah et al., 2008).

Les différences des teneurs en minéraux sont liées à l'origine diverse des légumes feuilles (Agbo et al., 2009). Les légumes feuilles présents sur les marchés pourraient provenir d'autres sites, jardins familiaux et de champs.

Les teneurs en potassium des légumes feuilles montrent qu'il y a une différence significative au seuil de 5\% entre les sites de collectes. Les légumes feuilles sont riches en potassium et c'est au niveau des marchés que les teneurs sont plus élevées (22403 mg / 100 g de MS pour la célosie du marché Abobogare contre $4477 \mathrm{mg} / 100 \mathrm{~g}$ de MS de la célosie du site maraîcher) (Tableau 6). L'amarante (12861 mg / $100 \mathrm{~g}$ de MS), la morelle (9262 mg / $100 \mathrm{~g}$ de MS) et le caya blanc (10745 mg /100 g de MS) du marché Gouro et la célosie (22403 mg / 100 g de MS), la corête potagère $(6792 \mathrm{mg} / 100 \mathrm{~g}$ de MS) du marché abobo gare ont les teneurs en potassium élevées que ceux du site. Les légumes feuilles sont une bonne source de potassium, surtout qu'il joue un rôle dans la contraction du muscle squelettique (Mensah et al., 2008).
Les teneurs en magnesium sont autant élevées au niveau des marchés et site maraîcher, avec 1947 mg / 100 g de MS (amarante marché Gouro) contre $1820 \mathrm{mg} /$ $100 \mathrm{~g}$ de MS (amarante du site maraîcher) (Tableau 7) qui supérieure à celle trouvée par Odhav et al. (2007) pour l'amarante (1317 $\mathrm{mg}$ ).

Les teneurs en magnésium des légumes feuilles montrent qu'il ya une différence significative au seuil de 5\% entre les marchés et le site maraîcher. Il n'y a pas de différence significative au niveau de la teneur en magnésium de la morelle du site et le marché Gouro, ainsi la corête Potagère du marché gouro et abobo gare. La morelle $(682 \mathrm{mg} /$ $100 \mathrm{~g}$ de MS), le caya blanc $1508 \mathrm{mg} / 100 \mathrm{~g}$ de MS du site maraîcher ont les teneurs en magnésium élevées que celles des marchés. Ces différences sont influencées par les techniques culturales (Nangula et al., 2010). $\mathrm{Au}$ niveau du site maraîcher, les producteurs utilisent beaucoup l'engrais chimique de type NPK, quant aux marchés les légumes feuilles pourraient provenir des jardins familiaux, d'autres sites et champs.

Les teneurs en fer des légumes feuilles montrent qu'il y a une différence significative au seuil de $5 \%$ entre les marchés et site maraîcher. L'amarante (56 mg / $100 \mathrm{~g}$ de MS), la célosie (44 mg / 100 g de MS) du marché abobo gare, la morelle (74 mg / $100 \mathrm{~g}$ de MS), le caya blanc (52 mg / 100 g de MS) du marché Gouro ont les teneurs en fer plus élevées que celles du site maraîcher (Tableau 8).

Les valeurs en fer mentionnées en comparaison avec celles obtenues par Singh et al. (2001) (amarante = 7,6 mg), Grubben et al. (2004) (corête $=7,2 \mathrm{mg}$ et célosie $=7,8 \mathrm{mg}$ ) et Akubugwo et al. (2007) (morelle $=13,01 \mathrm{mg}$ ) montrent beaucoup de différences. Cependant les légumes feuilles sont une bonne source de fer qui pourrait aider au recouvrement d'un problème nutritionnel comme l'anémie et d'autres déficiences en micronutriments (Lyimo et al., 2003; Nangula et al., 2010 ). 
Tableau 1 : Teneur en sucres totaux des légumes feuilles en g / $100 \mathrm{~g}$ de MS.

\begin{tabular}{lccc}
\hline & $\begin{array}{l}\text { Site } \\
\text { Maraîcher } \\
\text { (Port-Bouet) }\end{array}$ & Marché Gouro & Marché Abobo gare \\
\hline Amaranthus hybridus & $0,44 \pm 0,00^{\mathrm{a}}$ & $0,65 \pm 0,12^{\mathrm{c}}$ & $0,56 \pm 0,11^{\mathrm{b}}$ \\
Solanum nigrum & $0,74 \pm 0,01^{\mathrm{a}}$ & $1,21 \pm 0,35^{\mathrm{b}}$ & $1,38 \pm 0,02^{\mathrm{c}}$ \\
Corchorus olitorius $_{\text {Celosia argentea }}$ & $0,67 \pm 0,00^{\mathrm{b}}$ & $1,16 \pm 0,46^{\mathrm{c}}$ & $0,52 \pm 0,01^{\mathrm{a}}$ \\
Cleome gynandra & $0,67 \pm 0,00^{\mathrm{a}}$ & $0,83 \pm 0,31^{\mathrm{b}}$ & $0,66 \pm 0,01^{\mathrm{a}}$ \\
\hline Les chiffres en ligne ayant les mêmes lettres ne sont pas significativement différents au seuil de 5\%.
\end{tabular}

Tableau 2 : Teneur en sucres réducteurs des légumes feuilles en g/ $100 \mathrm{~g}$ de MS.

\begin{tabular}{lccc}
\hline & $\begin{array}{l}\text { Site Maraîcher } \\
\text { (Port-Bouet) }\end{array}$ & Marché Gouro & Marché Abobo gare \\
\hline Amaranthus hybridus & $0,14 \pm 0,00^{\mathrm{b}}$ & $0,07 \pm 0,00^{\mathrm{a}}$ & $0,07 \pm 0,00^{\mathrm{a}}$ \\
Solanum nigrum & $0,09 \pm 0,00^{\mathrm{b}}$ & $0,07 \pm 0,0^{\mathrm{a}}$ & $0,09 \pm 0,00^{\mathrm{b}}$ \\
Corchorus olitorius $_{\text {Celosia argentea }}$ & $0,17 \pm 0,00^{\mathrm{c}}$ & $0,26 \pm 0,00^{\mathrm{b}}$ & $0,08 \pm 0,00^{\mathrm{a}}$ \\
Cleome gynandra & $0,08 \pm 0,00^{\mathrm{a}}$ & $0,07 \pm 0,00^{\mathrm{a}}$ & $0,07 \pm 0,002^{\mathrm{a}}$ \\
\hline
\end{tabular}

Les chiffres en ligne ayant les mêmes lettres ne sont pas significativement différents au seuil de $5 \%$.

Tableau 3: Teneur en protéine des legumes feuilles en $\%$.

\begin{tabular}{lcrc}
\hline & $\begin{array}{l}\text { Site Maraîcher } \\
\text { (Port-Bouet) }\end{array}$ & Marché Gouro & Marché Abobo gare \\
\hline Amaranthus hybridus & $33,32 \pm 0,03^{\mathrm{c}}$ & $35,41 \pm 0,05^{\mathrm{c}}$ & $37,21 \pm 0,15^{\mathrm{c}}$ \\
Solanum nigrum & $29,9 \pm 0,05^{\mathrm{b}}$ & $37,58 \pm 0,21^{\mathrm{c}}$ & $34,43 \pm 0,02^{\mathrm{b}}$ \\
Corchorus olitorius $^{\mathrm{b}}$ & $24,89 \pm 0,23^{\mathrm{a}}$ & $19,67 \pm 0,51^{\mathrm{a}}$ & $32,18 \pm 0,01^{\mathrm{a}}$ \\
Celosia argentea $_{\text {Cleome gynandra }}$ & $34,13 \pm 0,37^{\mathrm{c}}$ & $32,84 \pm 0,04^{\mathrm{b}}$ & $34,25 \pm 0,03^{\mathrm{b}}$ \\
\hline Les chiffres en ligne ayant les mêmes lettres ne sont pas significativement différents au seuil de $5 \%$ & $33,58 \pm 0,06^{\mathrm{a}}$ \\
\hline
\end{tabular}

Les chiffres en ligne ayant les mêmes lettres ne sont pas significativement différents au seuil de $5 \%$.

Tableau 4: Teneur en phosphore des légumes feuilles en mg / $100 \mathrm{~g}$ de MS.

\begin{tabular}{lccc}
\hline & $\begin{array}{c}\text { Site Maraîcher } \\
\text { (Port-Bouet) }\end{array}$ & Marché Gouro & Marché Abobo gare \\
\hline Amaranthus hybridus & $2050 \pm 1,94^{\mathrm{c}}$ & $1981 \pm 15,17^{\mathrm{b}}$ & $1721 \pm 0,42^{\mathrm{b}}$ \\
Solanum nigrum & $158,42 \pm 2,02^{\mathrm{a}}$ & $1916 \pm 11,21^{\mathrm{b}}$ & $2229 \pm 12,15^{\mathrm{c}}$ \\
Corchorus olitorius & $2262 \pm 1,43^{\mathrm{e}}$ & $1464 \pm 15,67^{\mathrm{a}}$ & $166,31 \pm 6,17^{\mathrm{b}}$ \\
Celosia argentea & $1772 \pm 1,94^{\mathrm{b}}$ & $1517 \pm 10,74^{\mathrm{a}}$ & $1369 \pm 9,36^{\mathrm{a}}$ \\
Cleome gynandra & $2223 \pm 1,36^{\mathrm{d}}$ & $1452 \pm 0,16^{\mathrm{a}}$ & $2410 \pm 13,07^{\mathrm{d}}$ \\
\hline \multicolumn{4}{l}{ Les chiffres en ligne ayant les mêmes lettres ne sont pas significativement différents au seuil de 5\%. }
\end{tabular}

Les chiffres en ligne ayant les mêmes lettres ne sont pas significativement différents au seuil de $5 \%$. 
Tableau 5: Teneur en calcium des légumes feuilles en mg / $100 \mathrm{~g}$ de MS.

\begin{tabular}{lccc}
\hline & $\begin{array}{c}\text { Site Maraîcher } \\
\text { (Port-Bouet) }\end{array}$ & Marché Gouro & Marché Abobo gare \\
\hline Amaranthus hybridus & $2893 \pm 1,44^{\mathrm{d}}$ & $5663 \pm 2,18^{\mathrm{c}}$ & $1901 \pm 0,35^{\mathrm{a}}$ \\
Solanum nigrum & $1509 \pm 0,90^{\mathrm{a}}$ & $1784 \pm 0,720^{\mathrm{b}}$ & $2260 \pm 0,38^{\mathrm{b}}$ \\
Corchorus olitorius $_{\text {Celosia argentea }}$ & $2489 \pm 0,73^{\mathrm{c}}$ & $1546 \pm 0,87^{\mathrm{a}}$ & $2159 \pm 0,75^{\mathrm{b}}$ \\
Cleome gynandra & $1981 \pm 0,62^{\mathrm{b}}$ & $1986 \pm 0,51^{\mathrm{b}}$ & $2635 \pm 1,00^{\mathrm{c}}$ \\
\hline
\end{tabular}

Les chiffres en ligne ayant les mêmes lettres ne sont pas significativement différents au seuil de $5 \%$.

Tableau 6: Teneur en potassium des légumes feuilles en mg / 100 g de MS.

\begin{tabular}{lccc}
\hline & $\begin{array}{l}\text { Site Maraîcher } \\
\text { (Port-Bouet) }\end{array}$ & Marché Gouro & Marché Abobo gare \\
\hline Amaranthus hybridus & $7887 \pm 12,00^{\mathrm{c}}$ & $12861 \pm 3,81^{\mathrm{e}}$ & $9182 \pm 1,96^{\mathrm{c}}$ \\
Solanum nigrum & $4985 \pm 1,9^{\mathrm{ab}}$ & $9262 \pm 3,34^{\mathrm{c}}$ & $6633 \pm 0,58^{\mathrm{b}}$ \\
Corchorus olitorius & $5621 \pm 5,58^{\mathrm{b}}$ & $5115 \pm 2,68^{\mathrm{b}}$ & $6792 \pm 0,58^{\mathrm{b}}$ \\
Celosia argentea & $4477 \pm 2,22^{\mathrm{ab}}$ & $4076 \pm 1,21^{\mathrm{a}}$ & $22403 \pm 10,54^{\mathrm{d}}$ \\
Cleome gynandra & $5896 \pm 1,26^{\mathrm{b}}$ & $10745 \pm 2,86^{\mathrm{d}}$ & $3034 \pm 1,46^{\mathrm{a}}$ \\
\hline \multicolumn{2}{l}{ Les chiffres en ligne ayant les mêmes lettres ne sont pas significativement différents au seuil de 5\%. }
\end{tabular}

Tableau 7: Teneur en magnésium des légumes feuilles en mg / 100 g de MS.

\begin{tabular}{lccc}
\hline & $\begin{array}{c}\text { Site Maraîcher } \\
\text { (Port-Bouet) }\end{array}$ & Marché Gouro & Marché Abobo gare \\
\hline Amaranthus hybridus & $1820 \pm 0,16^{\mathrm{d}}$ & $1947 \pm 0,23^{\mathrm{e}}$ & $727 \pm 0,07^{\mathrm{c}}$ \\
Solanum nigrum & $682 \pm 0,02^{\mathrm{a}}$ & $628 \pm 0,04^{\mathrm{a}}$ & $394 \pm 0,12^{\mathrm{a}}$ \\
Corchorus olitorius $_{\text {Celosia argentea }}$ & $644 \pm 0,04^{\mathrm{a}}$ & $753 \pm 0,04^{\mathrm{b}}$ & $781 \pm 0,01^{\mathrm{d}}$ \\
Cleome gynandra & $1279 \pm 0,05^{\mathrm{b}}$ & $1332 \pm 0,16^{\mathrm{c}}$ & $1866 \pm 0,25^{\mathrm{e}}$ \\
\hline \multicolumn{2}{l}{ Les chiffres en ligne ayant les mêmes lettres ne sont pas significativement différents au seuil de $5 \%}$.
\end{tabular}

Les chiffres en ligne ayant les mêmes lettres ne sont pas significativement différents au seuil de $5 \%$.

Tableau 8: Teneur en Fer des légumes feuilles en mg / 100 g de MS.

\begin{tabular}{lccc}
\hline & $\begin{array}{c}\text { Site Maraîcher (Port- } \\
\text { Bouet) }\end{array}$ & Marché Gouro & $\begin{array}{c}\text { Marché Abobo } \\
\text { gare }\end{array}$ \\
\hline Amaranthus hybridus & $09 \pm 0,01^{\mathrm{a}}$ & $24 \pm 0,01^{\mathrm{a}}$ & $56 \pm 0,01^{\mathrm{e}}$ \\
Solanum nigrum & $15 \pm 0,01^{\mathrm{b}}$ & $74 \pm 0,01^{\mathrm{d}}$ & $34 \pm 0,01^{\mathrm{c}}$ \\
Corchorus olitorius $_{\text {Celosia argentea }}$ & $32 \pm 0,01^{\mathrm{c}}$ & $27 \pm 0,01^{\mathrm{b}}$ & $09 \pm 0,01^{\mathrm{a}}$ \\
Cleome gynandra & $33 \pm 0,01^{\mathrm{c}}$ & $27 \pm 0,00^{\mathrm{b}}$ & $44 \pm 0,01^{\mathrm{d}}$ \\
\hline
\end{tabular}

Les chiffres en ligne ayant les mêmes lettres ne sont pas significativement différents au seuil de 5\%. 


\section{Conclusion}

Les légumes feuilles ont un apport nutritionnel important, du fait de leur teneur en protéine et en matière minérale. Les teneurs en nutriments sont plus élevées au niveau des marchés que celles du site maraîcher. Au marché l'on retrouve tous les légumes feuilles provenant de site maraîcher, jardins familiaux et champs. Cette variabilité, nous a permis de constater qu'au niveau du marché Abobo-gare, les légumes feuilles étudiés ont les concentrations en nutriments les plus élevées et que la morelle noire constitue le légume feuille le plus riche en nutriments (protéine, fer, phosphore). Par ailleurs, il serait opportun d'encourager la population à la consommation des légumes feuilles car ils contribuent de façon significative à la santé et à la sécurité alimentaire des populations par leur haute valeur nutritive. Ils permettent aussi d'éviter certaines carences nutritionnelles et en particulier l'anémie.

\section{REMERCIEMENTS}

Les auteurs tiennent à remercier le Centre National de Recherche Agronomique (CNRA), les maraîchers et vendeuses des différentes communes pour leur collaboration.

\section{REFERENCES}

Agbo E, Kouamé C, Mahyao A, N'Zi JC, Fondio L. 2009. Nutritional importance of Indigenous Leafy Vegetables in Côte d'Ivoire. Acta Hort. 806, ISHS, 1 : 361366.

Akubugwo IE., Obasi AN, Ginika SC. 2007. Nutritional Potential of the Leaves and Seeds of Black Nightshade- Solanum nigrum L. Var virginicum from AfikpoNigeria. Pakistan J. Nut., 6(4): 323-326

ANONYME. 2001. Echantillonnage de fruits et légumes frais à des fins d'analyse en laboratoire. Agence Canadienne d'Inspection des Aliments, p.8.

AOAC. 1984. Official Methods of Analysis $\left(14^{\text {th }}\right.$ edn). Association of Official Chemists: Arlington, VA; 11.
AOAC. 2005. Phosphorus in Fruits and Fruit Product. Spectrophotometric Molybdovanadate Method (18 ${ }^{\text {th }}$ Edn). Association of Official Chemists: Arlington, VA; 89.

Audigie CL, Figarella, Zonzain. 1977. Manipulation d'Analyses Biochimiques. Do in édition : paris ; 274.

Bernfield P. 1955. Amylase $\alpha$ and $\beta$ methods. In Enzymology I ( $9^{\text {th }}$ édn), Colowich SP, Kaplan NO (eds). Academic Inc.: New York; 149-154.

Dubois M, Gilles KA, Hamilton JK, Roben FA, Smith F. 1956. Colorimetric method for determination of sugar and related substances. Anal. Chem., 28: 350-356.

Grubben GJH, Denton OA. 2004. Ressources Végétales de l'Afrique Tropicale: Légumes (Vol. 2). Fondation PROTA Backhuys Publishers: Wageningen Leyden ; 737.

Iheanacho, Kizito ME, Udebuani, Angela C. 2009. Nutritional Composition of Some Leafy Vegetables Consumed in Imo State, Nigeria. J. Appl. Sci. Environ. Manage., 13(3): 35-38.

Jansen van Rensburg WS, Venter SL, Netshiluvhi TR, Ven Der Heever E, Vorster HJ, JA de Ronde. 2004. Role of indigenous leafy vegetables in Combatting Hunger and Malnutrition. South Afr. J. Bot., 70(1): 52-59.

Kahane R, Temple L, Brat P, Bon H. 2005. Les légumes feuilles des pays tropicaux : diversité, richesse économique et valeur santé dans un contexte très fragile. Colloque Angers, p. 10.

Lyimo M, Temu RPC, Mugula JK. 2003. Identification and nutrient composition of indigenous vegetables of Tanzania. Pl. F. Hum. Nut., 58: 85-92.

Mensah JK, Okoli RI, Ohaju-Obodo JO, Eifediyi K. 2008. Phytochemical, nutritional and medical properties of some leafy vegetables consumed by Edo people of Nigeria. Afric. J. Biot., 7(14): 2304-2309. 
Nangula P Uusiku, Andre' Oelofse , Kwaku G Duodu, Megan JB, Mieke Faber. 2010. Nutritional value of leafy vegetables of sub-Saharan Africa and their potential contribution to human health: A review. J. F. Comp. Anal., 23: 499-509.

Nordeide MB, Hatloy A, Folling M, Lied E, Oshaug A. 1996. Nutrient composition and nutritional importance of green leaves and wild food resources in an agricultural district, Koutiala, in southern Mali. Int. J. F. Sce. Nut., 47: 455-478.

Odhav B, Beekrum S, Akula Us, Baijnath H. 2007. Preliminary assessment of nutritional value of traditional leafy vegetables in KwaZulu-Natal, South Africa. J. F. Comp. Anal., 20: 430-435. Sheetal Gupta, A Jyothi Lakshmi, MN Manjunath, Jamuna Prakash. 2005. Analysis of nutrient and antinutrient content of underutilized green leafy vegetables. $L W T$, 38: 339-345

Singh G, Asha Kawatra, Sehgal S. 2001. Nutritional composition of selected green leafy vegetables, herbs and carrots. Pl. F. Hum. Nut., 56: 359-364

Tchiegang C, Kitikil A. 2004. Données ethno nutritionnelles et caractéristiques physico-chimiques des légumes feuilles consommés dans la savane de l'Adamaoua (Cameroun). Tropicultura, 22(1): 11-18. 